\title{
Unified Point Selection and Surface-Based Registration Using a Particle Filter
}

\author{
Burton Ma and Randy E. Ellis \\ School of Computing, Queen's University at Kingston, Canada K7L 3N6 \\ mab@cs.queensu.ca, ellis@cs.queensu.ca
}

\begin{abstract}
We propose an algorithm for jointly performing registration point selection and interactive, rigid, surface-based registration. The registration is computed using a particle filter that outputs a sampled representation of the distribution of the registration parameters. The distribution is propagated through a point selection algorithm derived from a stiffness model of surface-based registration, allowing the selection algorithm to incorporate knowledge of the uncertainties in the registration parameters. We show that the behavior of target registration error improves as the quality measure of the registration points increases.
\end{abstract}

\section{Introduction}

One method of registering a patient to preoperative 3D medical images for use in imageguided surgery is to digitize anatomical registration points and match them to surface models derived from the images. A problem that has gone largely unexplored in this framework is how to intraoperatively guide the surgeon to regions of the anatomy that might contain good registration points.

A few selection algorithms have been described that could be applied to preoperatively choosing registration point sets. Simon [13] proposed to choose surface model points that maximized the noise amplification index [11] of a $6 \times 6$ scatter matrix. Gelfand et al. [2] used the condition number of Simon's scatter matrix to optimize ICP registration stability when aligning pairs of surfaces with significant overlap. Uniform sampling of the sphere of normal vectors was proposed by Rusinkiewicz and Levoy [12] as a method of point selection for registering pairs of range images. We [9] showed that Simon's scatter matrix was equivalent to the well known spatial-stiffness matrix of an unactuated, kinematically unconstrained elastic mechanism; we proposed a pointselection algorithm based on the coordinate frame invariant analysis of Lin et al. [8].

The problem with applying these kinds of selection algorithms to online guidance is that they all attempt to optimize some criteria based on points and surface normals defined in the model coordinate frame, but these features are uncertain during the course of a registration. If the true registration was known, then the model features could be inferred by finding the model points that corresponded to the patient registration points. One possible solution is to use the current set of $N$ patient points and a registration algorithm to estimate the corresponding model points, and then use the selection algorithm to suggest the next best model registration point. A possibly superior approach is to use a registration algorithm that estimates the uncertainties of the registration parameters 
and propagates the uncertainties through the selection process. If the algorithm represents the uncertainties as a covariance matrix, for instance Lavallée et al. [7], then the uncertainties can be propagated as a finite set of estimates of the registration parameters using a technique such as the unscented transform of Julier and Uhlmann [6].

In this article we unify our point selection algorithm [9] with our sampling-based registration algorithm [10] to produce a filter-selection algorithm that estimates the distribution of the registration parameters (instead of only the covariance) and computes which regions of the model are likely to contain good registration points.

\section{Stiffness-Based Point Selection}

We [9] proposed an algorithm for sequentially constructing a set of model registration points by greedily maximizing a quality measure derived from a stiffness model of shape-based registration. Our approach considers the $N$ registration points $\mathbf{p}_{i}$ to be the attachment locations for unloaded linear springs with orientations given by the surface normal vectors $\mathbf{n}_{i}$. This configuration of springs leads to a stiffness relationship

$$
\begin{aligned}
& \mathbf{w}=\mathbf{K t} \\
& \begin{array}{c}
\text { force } \\
\text { torque }
\end{array}\left[\begin{array}{c}
\mathbf{f} \\
\boldsymbol{\tau}
\end{array}\right]=\left(\sum_{i=1}^{N}\left[\begin{array}{c}
\mathbf{n}_{i} \\
\mathbf{p}_{i} \times \mathbf{n}_{i}
\end{array}\right]\left[\begin{array}{c}
\mathbf{n}_{i} \\
\mathbf{p}_{i} \times \mathbf{n}_{i}
\end{array}\right]^{T}\right)\left[\begin{array}{l}
\boldsymbol{v} \\
\boldsymbol{\omega}
\end{array}\right] \begin{array}{c}
\text { linear displacement } \\
\text { rotational displacement }
\end{array}
\end{aligned}
$$

where $\mathbf{w}$ is the wrench representing the reaction force and torque, and $\mathbf{t}$ is the twist representing the applied linear and rotational displacement.

Simon [13] analyzed $\mathbf{K}$ by examining its singular values, which are not frame invariant and are not directly comparable to one another because rotational and translational displacements have differing units. Frame-invariant quantities, called the principal translational and rotational stiffnesses, can be calculated from $\mathbf{K}$ (Lin et al. [8]); moreover, by considering a task-specific point target, the rotational stiffnesses can be scaled so that they are directly comparable to the translational stiffnesses. The scaled stiffnesses are called the equivalent rotational stiffnesses, and a stiffness quality measure is $Q=\min \left(\mu_{\mathrm{eq}, 1}, \mu_{\mathrm{eq}, 2}, \mu_{\mathrm{eq}, 3}, \sigma_{1}, \sigma_{2}, \sigma_{3}\right)$ where the $\mu_{\mathrm{eq}, i}$ are the equivalent rotational stiffnesses and the $\sigma_{i}$ are the translational stiffnesses. $Q$ characterizes the least constrained displacement of the mechanism; maximizing $Q$ will minimize the worstcase displacement of the mechanism. Our algorithm [9] takes as input a set of $N$ model registration points $\mathbf{p}_{i}$ with normal vectors $\mathbf{n}_{i}$ and a surface model from which to select points. The quality measure $Q$ is calculated and heuristics are used to find the model point $\mathbf{p}_{N+1}$ that maximizes the increase in $Q$. At least six point/normal vector pairs are required by the selection algorithm because $\mathbf{K}$ does not have full rank otherwise.

\section{Distribution of Registration Parameters}

We [10] proposed an algorithm that used a particle filter described by van der Merwe et al. [15] to estimate the distribution of the registration parameters. The registration 
parameters are represented by a state vector $\mathbf{x}_{t}=\left[\theta \phi \delta d_{x} d_{y} d_{z}\right]_{t}^{T}$ where $\theta, \phi$, and $\delta$ are the three rotation parameters, $d x, d y$, and $d z$ are the three translation parameters, and $t$ is the time index equal to the current number of registration points processed. The patient registration point acquired at time $t$ is treated as a control input $\mathbf{u}_{t}$. The state space model we used was

$$
\begin{aligned}
& \mathbf{x}_{t+1}= \mathbf{x}_{t}+\mathcal{N}\left(\mathbf{0}, \mathbf{Q}_{t}\right) \\
& \mathbf{y}_{t}=\left[\begin{array}{c}
\mathbf{r}\left(\theta_{t}, \phi_{t}, \delta_{t}\right)\left(\mathbf{u}_{1}+\left[d x_{t} d y_{t} d z_{t}\right]^{T}\right) \\
\vdots \\
\mathbf{r}\left(\theta_{t}, \phi_{t}, \delta_{t}\right)\left(\mathbf{u}_{t}+\left[d x_{t} d y_{t} d z_{t}\right]^{T}\right)
\end{array}\right]+\mathcal{N}\left(\mathbf{0}, \mathbf{R}_{t}\right)
\end{aligned}
$$

Equation 2 is called the process model and it describes how the state evolves in one time step; the model has a time-invariant state, except for the additive process noise, because the registration state is constant. The zero-mean Gaussian process noise with covariance $\mathbf{Q}_{t}$ allows the filter to move from initial estimates of the state to successively better estimates. $\mathbf{Q}_{t}$ is annealed towards $\mathbf{0}$ over time as the estimates become better.

The observation model is given by Equation 3 where $\mathbf{r}\left(\theta_{t}, \phi_{t}, \delta_{t}\right)$ is a rotation about a remote center and $\left[\begin{array}{lll}d x_{t} & d y_{t} & d z_{t}\end{array}\right]^{T}$ is a translation vector. We use rotational parameters that surgeons are most familiar with, measured as order-independent angles of rotation in the coronal, sagittal, and transverse planes. The form of the rotation matrix $\mathbf{r}(\theta, \phi, \delta)$ can be found in Iyun et al. [5]. In this article, we consider $\left[\begin{array}{lll}d x_{t} & d y_{t} & d z_{t}\end{array}\right]^{T}$ to be the translation to a remote center of rotation. The model is simply the estimated registration transformation applied to the patient registration points concatenated into a single vector; the length of the vector at time $t$ is $3 t$. We assume additive, zero-mean Gaussian noise with covariance $\mathbf{R}_{t}$; the noise is the displacement of each transformed registration point to the surface of the model. Like the process noise covariance $\mathbf{Q}_{t}$, the measurement noise covariance $\mathbf{R}_{t}$ must be annealed.

The output of the particle filter is a set of $P$ equally weighted samples (particles) representing the posterior distribution of the registration parameters. T o conserve space in this article we refer the reader to the article by van der Merwe et al. [15] for details on the mechanics of the specific particle filter we use. Note that the particle filter is not restricted to using Gaussian noises.

\subsection{Establishing an Initial Distribution}

A prior distribution $\mathbf{x}_{0}$ needs to be specified for the particle filter. Strictly speaking, the prior is supposed to be independent of the observations. A non-informative prior (a uniform distribution over the 6-dimensional state space) is impractical because an unwieldy number of particles would be required to adequately sample the space. We take a pragmatic approach and estimate an initial distribution using the first four registration points. The first registration point is used to estimate the translation to a remote center of rotation and the remaining three points are used to estimate the rotation. We assume that each of the first four points comes from a predefined region of the accessible surface of the bone. A small number of samples $s_{1}, s_{2}, s_{3}$, and $s_{4}$ are drawn from the four 

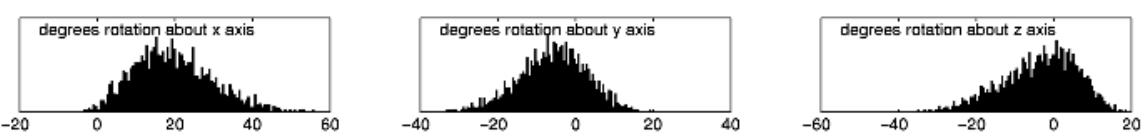

Fig. 1. Distributions of rotation parameters $(\theta, \phi$, and $\delta$ from left to right) obtained using the first four registration points

regions and the patient registration points are registered to all $s_{1} \cdot s_{2} \cdot s_{3} \cdot s_{4}$ configurations of model registration points. This sets the number of particles as $P=s_{1} \cdot s_{2} \cdot s_{3} \cdot s_{4}$ and yields an initial distribution from which we can start the filter. The resulting distributions (see example in Figure 1) are surprisingly wide in the range of rotation parameters.

\section{A Unified Filter-Selection Algorithm}

Our filter-selection algorithm uses the output of a particle filter registration algorithm to propagate the distribution of registration parameters through a point selection process. Each of the $P$ registration estimates (particles) is applied to the set of patient registration points and the corresponding model points are inferred by performing a nearest neighbor calculation; correspondences weighted by distance (for instance, [1], [3], or [4]) could also be used. Each of the $P$ sets of model correspondences is used as input to the point selection algorithm that calculates the increase $\delta Q$ in quality measure $Q$ for each of the $M$ model points. This calculation requires $O(P M)$ time and is the major drawback of this algorithm. If we stored the distribution of $\delta Q$ for each model point then we would also require $O(P M)$ storage; we compute only the mean increase $\Delta Q$ for each model point which requires $O(M)$ storage.

If we examine the model points with mean quality measure higher than a certain value - for example, the $95^{\text {th }}$ percentile - then what we find is that those model points tend to come from one or more continuous regions of the model. This presents the surgeon with the task of locating any point inside a region, which is much easier than trying to locate a single point. Examples of point-selection regions are shown in Figure 2. The unified filter-selection algorithm is shown in Figure 3

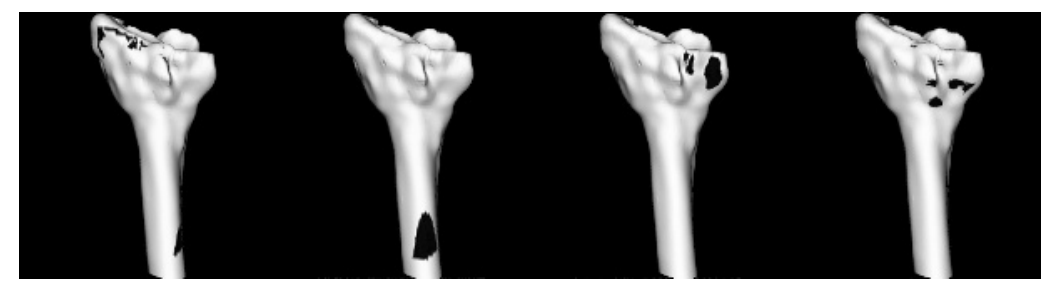

Fig. 2. Regions (in black) with mean quality measure greater than the $95^{\text {th }}$ percentile after $7,8,9$, and 10 (from left to right) registration points have been processed using the algorithm described in Figure 3 
- Digitize 6 registration points $\mathbf{U}$ so that the stiffness matrix $\mathbf{K}$ has full rank.

- For $i=6,7, \ldots, N$ (main loop)

- Update the particle filter to obtain $P$ registration samples $\left\{\mathbf{x}_{1}, \ldots, \mathbf{x}_{P}\right\}$.

- Initialize $\Delta Q[1 . . M]=0$ where $M$ is the number of model points. $\Delta Q[m]$ is the mean increase in the quality measure for the $m^{\text {th }}$ model point, where the mean is calculated over the $P$ particles.

- For $j=1,2, \ldots, P$

* Apply the estimated registration transformation to $\mathbf{U}: \mathbf{Y}=\mathbf{T}\left(\mathbf{x}_{j}\right) \mathbf{U}$.

* Find the model points $\mathbf{P}$ closest to $\mathbf{Y}$.

* Compute the quality measure $Q$ of $\mathbf{P}$.

* For $k=1,2, \ldots, M$

- $\mathbf{p}_{k}=k^{\text {th }}$ model point

- $\mathbf{n}_{k}=$ normal vector at $\mathbf{p}_{k}$

$\Delta Q[k]=\Delta Q[k]+\delta Q\left(\mathbf{p}_{k}, \mathbf{n}_{k}\right) / P$ where $\delta Q\left(\mathbf{p}_{k}, \mathbf{n}_{k}\right)$ is an approximation of the increase in quality measure if point $\mathbf{p}_{k}$ with normal $\mathbf{n}_{k}$ is added to the current set of estimated model registration points $\mathbf{P}$; see [9] for details.

- Find the model points with $\Delta Q$ in the range $[l o, h i]$ (lo and $h i$ might be the $95^{\text {th }}$ and $100^{\text {th }}$ percentiles, for example) defining regions on the model that contain good registration points. Digitize a patient point $\mathbf{u}_{i+1}$ corresponding to a point inside these regions.

- $\mathbf{U}=\left\{\mathbf{U}, \mathbf{u}_{i+1}\right\}$.

Fig. 3. A filter-selection algorithm

\section{Materials and Methods}

Bone-surface models of a distal radius, proximal femur, and proximal tibia were used to validate the proposed filter-selection algorithm. The models were derived from CT scans of patient volunteers. The surgically accessible area and a target were defined on each model. These features are shown in Figure 4. Four regions were defined on each phantom from where the initial four points needed to initialize the particle filter were drawn from; an additional two large regions were defined on each phantom to yield the requisite six points for the selection phase of the algorithm. The number of particles used was $7^{4}=2401$ for the radius and tibia, and $8 \times 7^{3}=2744$ for the femur. The simulations were run on a PC with an AMD Athlon $2400 \mathrm{CPU}$ and 256MB of RAM.

Registration simulations were run using each model with 200 trials, each trial executing the steps shown in Figure 5. The total number of registration points $N$ was
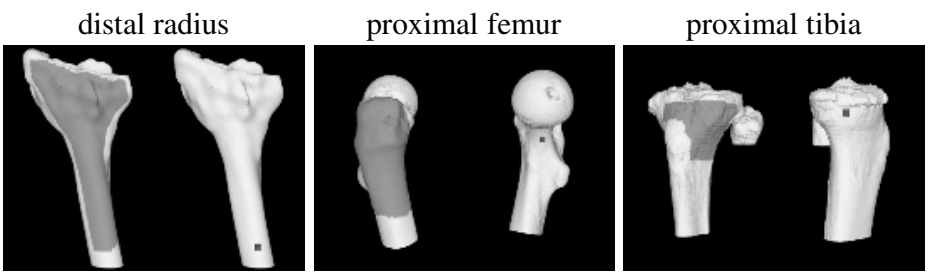

Fig. 4. Features used in the experimental validation of integrated point selection and registration. Accessible regions are shown in gray and targets are shown as cubes. 
1. Randomly select registration points from the six predefined regions; $\mathbf{U}=\left\{\mathbf{u}_{1}, \mathbf{u}_{1}, \ldots, \mathbf{u}_{6}\right\}$.

2. Add Gaussian noise $\mathcal{N}\left(0,(0.35 \mathrm{~mm})^{2}\right)$ to the $x, y$, and $z$ components of the points in $\mathbf{U}$.

3. Displace $\mathbf{U}$ by the inverse of the randomly selected registration transformation.

4. Calculate the initial state distribution (Section 3.1).

5. For $i=6 . . N$

(a) Apply one iteration of the main loop of the filter-selection algorithm (Figure 3 .

(b) Add Gaussian noise drawn from $\mathcal{N}\left(0,(0.35 \mathrm{~mm})^{2}\right)$ to the new registration point $\mathbf{u}_{i+1}$

Fig. 5. Simulation steps for the experiment described in Figure 3

20. We took $\mathbf{Q}_{t}$ to be uncorrelated with initial variances of $\left(3^{\circ}\right)^{2}$ and $(3 \mathrm{~mm})^{2}$ for the rotational and translational components. We took $\mathbf{R}_{t}$ to be uncorrelated with initial variances of $(2 \mathrm{~mm})^{2}$ in each of the $x, y$, and $z$ components of the observation vector. Because we used a relatively small number of registration points, the annealing factors were both chosen to be the relatively small value of 0.7 and we annealed for a total of 10 time steps. Three different ranges of $[l o, h i]$ were used to determine if point selection had an effect on registration precision and accuracy; the ranges used, in percentiles of $\delta Q$, were $[50,55],[75,80]$, and $[95,100]$. The component-wise median of the filter distribution was used as the estimate of the registration state.

\section{Results}

The mean of the 200 estimated registration states was within $0.2^{\circ}$ in each of the true rotation states, and within $0.1 \mathrm{~mm}$ of the true translation states; this was true regardless of the model used or the range of $[l o, h i]$. This strongly suggests that there is no bias in the filter estimates of registration. The standard deviations of the 200 estimated registration states are shown in Table 1 for each model and range of $[l o, h i]$. There was a trend towards decreasing variance as points that lead to increasing $\Delta Q$ values were used. This is more clearly seen in Figure 6 which shows the distributions of target registration error (TRE). There was almost no difference in the distribution of TRE between point sets with $\Delta Q$ values of $[50,55]$ and $[75,80]$. There was clear improvement in TRE

Table 1. Standard deviations of the registration state parameters

\begin{tabular}{|c|c|c|c|c|c|c|c|}
\hline Model & {$[$ lo, hi $]$} & $\theta_{x}$ & $\theta_{y}$ & $\theta_{z}$ & $t_{x}$ & $t_{y}$ & $t_{z}$ \\
\hline \multirow{3}{*}{ radius } & {$[50,55]$} & $0.57^{\circ}$ & $0.58^{\circ}$ & $1.57^{\circ}$ & $0.47 \mathrm{~mm}$ & $0.27 \mathrm{~mm}$ & $0.45 \mathrm{~mm}$ \\
& {$[75,80]$} & $0.50^{\circ}$ & $0.55^{\circ}$ & $1.55^{\circ}$ & $0.48 \mathrm{~mm}$ & $0.21 \mathrm{~mm}$ & $0.40 \mathrm{~mm}$ \\
& {$[95,100]$} & $0.38^{\circ}$ & $0.38^{\circ}$ & $1.22^{\circ}$ & $0.47 \mathrm{~mm}$ & $0.18 \mathrm{~mm}$ & $0.35 \mathrm{~mm}$ \\
\hline \multirow{3}{*}{ femur } & {$[50,55]$} & $0.95^{\circ}$ & $0.56^{\circ}$ & $1.32^{\circ}$ & $0.23 \mathrm{~mm}$ & $0.48 \mathrm{~mm}$ & $0.38 \mathrm{~mm}$ \\
& {$[75,80]$} & $0.70^{\circ}$ & $0.52^{\circ}$ & $1.22^{\circ}$ & $0.27 \mathrm{~mm}$ & $0.49 \mathrm{~mm}$ & $0.38 \mathrm{~mm}$ \\
& {$[95,100]$} & $0.87^{\circ}$ & $0.55^{\circ}$ & $0.91^{\circ}$ & $0.19 \mathrm{~mm}$ & $0.32 \mathrm{~mm}$ & $0.41 \mathrm{~mm}$ \\
\hline \multirow{3}{*}{ tibia } & {$[50,55]$} & $1.59^{\circ}$ & $0.82^{\circ}$ & $1.05^{\circ}$ & $0.45 \mathrm{~mm}$ & $0.36 \mathrm{~mm}$ & $0.79 \mathrm{~mm}$ \\
& {$[75,80]$} & $1.49^{\circ}$ & $0.87^{\circ}$ & $0.97^{\circ}$ & $0.47 \mathrm{~mm}$ & $0.40 \mathrm{~mm}$ & $0.64 \mathrm{~mm}$ \\
& {$[95,100]$} & $1.09^{\circ}$ & $0.71^{\circ}$ & $0.73^{\circ}$ & $0.34 \mathrm{~mm}$ & $0.32 \mathrm{~mm}$ & $0.51 \mathrm{~mm}$ \\
\hline
\end{tabular}


Distal radius
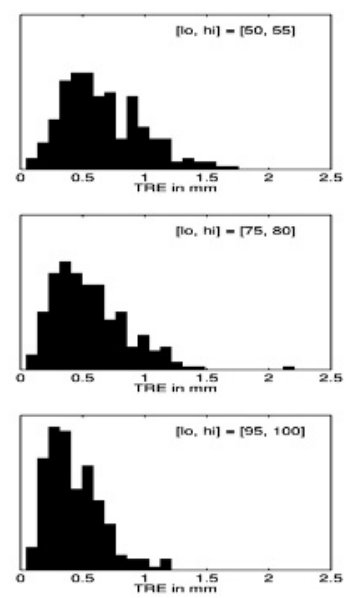

Proximal femur
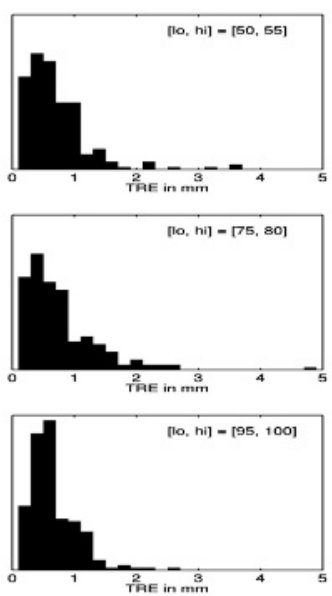

Proximal tibia

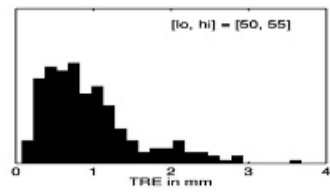

$[10$, hi] $-[75,80]$
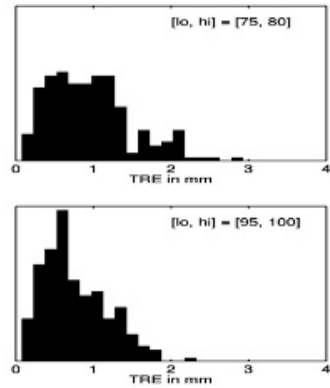

Fig. 6. Distribution of target registration errors over 200 trials for each model and range of $\Delta Q$ values $[l o, h i]$

when using the highest range, $[95,100]$, of $\Delta Q$ values; however, the actual decrease in expected TRE was very small. The computation time to process 20 registration points was between two and three minutes on a relatively low-cost PC.

\section{Discussion}

Estimating the translation to a remote center of rotation appears to be an effective statespace model for rigid registration. This produces a better initial distribution than the random sampling approach taken in [10]; we used fewer particles and obtained more accurate registrations. Granger and Pennec [3] noted that their EM-ICP registration algorithm was most sensitive to errors in the translational component of the initial transformation estimate. This appears to be true for the particle-filter registration method as well. Figure 1 shows that the range of the rotation prior is at least $60^{\circ}$ in each component for the tibia model. If this observation is true in general, then the initial distribution could be established using a single region to estimate the translation to the remote center of rotation; the rotation parameters could be estimated by having the surgeon hold a tracked target to approximately indicate the directions of the anatomic axes.

One feature of online point selection is that the surgeon is given a clear visualization of where good registration points are likely located. This may be an advantage when the surgeon has little experience performing registration, such as when attempting a particular procedure for the first time, or during adoption of image-guided surgery.

Both the particle filter and the point-selection algorithm can exploit parallel computation, and this would be one way to provide integrated point selection at an acceptable speed. A better approach might be to replace the particle filter with a more efficient type of filter. In particular, the family of Gaussian mixture sigma-point particle (GMSPP) filters described by van der Merwe and Wan [14] are reportedly much more efficient in 
terms of computation than particle filters. These filters represent the posterior using a Gaussian mixture model. Because there is a deterministic way to draw a small number of samples from a Gaussian distribution (for example, see Julier and Uhlmann [6]) that preserves all of the relevant information, only a small number of particles might need to be considered during point selection if a GMSPP filter is used.

This work shows that it is possible unify surface-based registration, optimal selection of the next point from the patient's anatomy, and estimation of the uncertainty in the resulting registration. Optimization of the algorithm will bring it into the timeframe required in orthopedic surgery. Extensions to this work might include application to deformable atlas-based registration and to volumetric registration of one image to another.

\section{References}

[1] H. Chui and A. Rangarajan. A feature registration framework using mixture models. In IEEE Workshop on Mathematical Methods in Biomedical Image Analysis, June 2000.

[2] N. Gelfand, L. Ikemoto, S. Rusinkiewicz, and M. Levoy. Geometrically stable sampling for the ICP algorithm. In 3DIM 2003, Banff, Canada, Oct 2003. IEEE.

[3] S. Granger and X. Pennec. Multi-scale EM-ICP: A fast and robust approach for surface registration. In ECCV 2002, pages 418-432, Copenhagen, Denmark, May 2002. Springer.

[4] W. E. L. Grimson, G. J. Ettinger, S. J. White, T. Lozano-Pérez, W. M. Wells III, and R. Kikinis. An automatic registration method for frameless stereotaxy, image guided surgery, and enhanced reality visualization. IEEE T Med Imaging, 15(2):129-140, 1996.

[5] O. Iyun, D. P. Borschneck, and R. E. Ellis. Computer-assisted correction of bone deformities. In MICCAI 2002, pages 232-240, Tokyo, Japan, 2002. Springer.

[6] S. J. Julier and J. K. Uhlmann. A general method for approximating nonlinear transformations of probability distributions. Technical report, Oxford University, 1996.

[7] S. Lavallée, R. Szeliski, and L. Brunie. Anatomy-based registration of three-dimensional medical images, range images, $\mathrm{x}$-ray projections, and three-dimensional models using octree-splines. In Computer-Integrated Surgery, pages 115-143. The MIT Press, 1996.

[8] Q. Lin, J. Burdick, and E. Rimon. A stiffness-based quality measure for compliant grasps and fixtures. IEEE T Robotic Autom, 16(6):675-688, 2000.

[9] B. Ma and R. E. Ellis. Spatial-stiffness analysis of surface-based registration. In MICCAI 2004, pages 623-630, St. Malo, France, 2004. Springer.

[10] B. Ma and R. E. Ellis. Surface-based registration with a particle filter. In MICCAI 2004, pages 566-573, St. Malo, France, 2004. Springer.

[11] A. Nahvi and J. M. Hollerbach. The noise amplification index for optimal pose selection in robot calibration. In IEEE ICRA, Minneapolis, Minnesota, Apr 1996.

[12] S. Rusinkiewicz and M. Levoy. Efficient variants of the ICP algorithm. In 3DIM 2001, pages 145-152, Québec City, Canada, May 2001. IEEE.

[13] D. A. Simon. Fast and Accurate Shape-Based Registration. PhD thesis, Carnegie Mellon University, Pittsburgh, Pennsylvania, Dec 1996.

[14] R. van der Merwe and E. Wan. Gaussian mixture sigma-point particle filters for sequential probabilistic inference in dynamic state-space models. In ICASSP, 2003.

[15] R. van der Merwe, A. Doucet, N. de Freitas, and E. Wan. The unscented particle filter. Technical Report CUED/F-INFENG/TR 380, Cambridge Univ. Dept. of Eng., 2000. 